\title{
Neurodevelopmental risk factors in schizophrenia
}

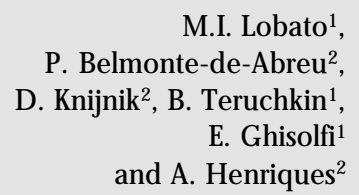

M.I. Lobato ${ }^{1}$, P. Belmonte-de-Abreu², D. Knijnik², B. Teruchkin ${ }^{1}$, E. Ghisolfi ${ }^{1}$ and $\mathrm{A}$. Henriques ${ }^{2}$

\author{
${ }^{1}$ Serviço de Psiquiatria, Hospital de Clínicas de Porto Alegre, \\ Porto Alegre, RS, Brasil \\ ${ }^{2}$ Departamento de Psiquiatria, Faculdade de Medicina, \\ Universidade Federal do Rio Grande do Sul, Porto Alegre, RS, Brasil
}

\section{Correspondence \\ M.I. Lobato \\ Rua Vasco da Gama, 1301/401 \\ 90420-112 Porto Alegre, RS \\ Brasil \\ E-mail: mlupo@pro.via-rs.com.br}

Research supported by CNPq (No. 138121/96-6).

Received August 11, 1999 Accepted November 22, 2000

\section{Abstract}

The authors review environmental and neurodevelopmental risk factors for schizophrenic disorders, with emphasis on minor physical anomalies, particularly craniofacial anomalies and dermatoglyphic variations. The high prevalence of these anomalies among schizophrenic subjects supports the neurodevelopmental theory of the etiology of schizophrenia, since they suggest either genetically or epigenetically controlled faulty embryonic development of structures of ectodermal origin like brain and skin. This may disturb neurodevelopment that in turn may cause these subjects to be at increased risk for the development of schizophrenia and related disorders. The precise confirmation of this theory, at least in some cases, will provide further understanding of these illnesses, allowing easy and inexpensive identification of subjects at risk and providing guidelines for the development of new pharmacological interventions for early treatment and even for primary prevention of the illness.

\section{Introduction}

Despite many technical and medical advances over the past ten years, at the end of the Decade of the Brain schizophrenia is still a personal and family disaster. Research over the past few decades has demonstrated with increasing frequency the presence of brain lesions dating back to early brain development (1). This has resulted in a change in the concept of schizophrenia from a disease of the mind to a disease of the brain. This neurodevelopmental model is not a new concept. In fact, it is a reconsideration of the XIX century's concept about a precocious psychotic condition preceding a "secondary dementia" (2) that affects adults from 18 to 24 years, particularly men, with associated cognitive impairment early in the course of the disease.

Here we review the main evidence for the neurodevelopmental theory of schizophrenia. The articles for this review were selected through a systematic literature search of Medline from 1994-1999. The key words used were "schizophrenia", "neurodevelopmental", and "risk factors". The criteria for selection of the articles were systematic assessment using DSM-III-IV criteria, casecontrol design, and adequate description of index and comparison cases. Additional papers from citations of these articles were also 
included according to the same criteria.

\section{Evidence for association of neurode- velopmental abnormalities with the course of schizophrenia}

Neurodevelopmental disorders such as speech retardation and reading and/or motor disabilities are usually non-progressive and appear during the first decade of life. Cerebral morphologic alterations include microcephaly, hydrocephalus, abnormal cell migration and asymmetry of specific brain regions (3). The schizophrenias have many characteristics of neurodevelopmental disorders, although, because of their many different clinical presentations, there is often great difficulty in making any general statement about this group of disorders. If we consider the neurodevelopmental theory in schizophrenia, it is reasonable to presume that the morbid process is present in a latent state and that the lesion(s) and some neurodevelopmental abnormalities could be observed before diagnosis. Although most prospective studies that have looked for neurodevelopmental abnormalities in schizophrenia before diagnosis report negative results, some did detect subtle behavior and intellectual abnormalities as possible "predictors of risk" for the development of schizophrenia (4). These findings, however, are inconsistent, raising doubts about the neurodevelopmental theory $(3,4)$.

A persistent question can be raised. If the initial lesion occurs in early neurodevelopment, why would the disease become clinically manifest usually at the end of adolescence? A possible explanation is the occurrence of some maturational processes throughout adolescence that modulate psychosis (2). It is known that the human brain reaches approximately adult size during the early school years, and that this growth seems to be affected by the increase of gray matter volume as opposed to white matter volume $(2,3,5)$. It is also known that the fine tuning of neuronal connections and the selective reduction of cerebral structures occurs during early cerebral maturation. A genetic alteration in some maturational mechanisms, such as "cortical pruning", expressed during adolescence can explain the delay between initial lesion and illness onset $(2,5)$. According to some authors (3), most schizophrenic patients display a clinical picture that fits the neurodegenerative model better than the neurodevelopmental model. It consists of a decline in function of previously healthy persons, progresses over months or years with exacerbations and remissions and higher or lower expression of psychotic and/or negative symptoms, with only a small group of patients returning to the premorbid state (3). Complete remission of schizophrenia is not often seen in patients diagnosed according to rigorous diagnostic criteria.

\section{Neuropathological evidence}

An important issue for the neurodevelopmental theory is the failure of major early studies to detect gliosis (considering gliosis as the evidence of a brain reaction to afterbirth insults, producing "glial scars") (3). Cerebral abnormalities in the absence of gliosis may probably indicate abnormalities of fetal cerebral development instead of neurodegeneration (3). Some neuropathological studies have failed to detect evidence of gliosis (1), but others have described it in subcortical and periventricular areas. During cerebral development, neuronal proliferation occurs in ventricular and subventricular zones, with cortical cells migrating through radially aligned glial fibers to the final positions. Therefore, reduction or derangement of the neurons in the cortical areas would be an indication of cerebral development abnormalities $(2,5)$.

There is evidence that schizophrenic brains are approximately 5-8\% lighter and $4 \%$ smaller than healthy controls $(2,6)$. Nelson et al. (7), in a magnetic resonance 
imaging (MRI) meta-analysis study, demonstrated a positive association between schizophrenia and the reduction of the bilateral hippocampus and, probably, amygdala. Akbarian et al. (8) found a reduction in neurons expressing NADPH-diaphorase in more external cortical layers and an excess in the inferior layers of white matter of the frontal and temporal cortex of schizophrenics. Several other studies of chronic institutionalized patients demonstrated a reduction of total cerebral weight, hemispheric volume, cortical volume and gray matter size, together with ventricular enlargement, suggesting that brain changes could be risk factors, rather than "causal" factors $(3,9)$. Gur et al. (10), using MRI measurements, found a reduction in gray matter volume in chronic and neuroleptic-naive schizophrenic patients, also indicating a developmental process. Ventricular enlargement is also found in several genetic and neurodevelopmental diseases, and in 15 to $30 \%$ of schizophrenic patients (3). Many studies have reported higher ventricular brain ratios in schizophrenic patients compared to controls (11), and there is also evidence that the enlargement is present before the onset of the disease and is not progressive (12).

According to Stevens (3) and others (9, 11 ), in spite of demonstrating smaller ventricular brain ratio differences than reported in previous studies, this finding still is of unquestionable value for the understanding of the disease and for the demonstration of disease heterogeneity.

\section{Chronological markers}

The chronological markers of neuroembryogenesis refer to anthropometric characteristics that reflect a prenatal insult. The most studied anthropometric markers in schizophrenia are minor physical anomalies (MPA) and dermatoglyphic marks (DM). Several other psychiatric disorders show association with MPA and DM (e.g., child- hood hyperkinetic syndrome, autism and behavioral childhood disturbances). MPA found in the cephalic area, hands, and feet can indicate a neurodevelopmental problem dating back to the first gestational quarter. DM consists of digital and palmar line alterations and indicates second-trimester developmental problems (13-17).

The rationale underlying the association of MPA/DM and disturbed neurogenesis is based on the fact that each of these organs originates from the same primordial tissue. The neural tube develops from the primitive ectoderm, with the ventricular system and cerebral tissues originating from the neural tube lumen. As a case in point, retinoic acid excess or deficit in the first trimester can simultaneously affect both neuronal migration and cortical subplate development and non-neuronal surface ectoderm formation (18). Such maldevelopment can result in craniofacial abnormalities, particularly of the eyes and ears, as well as surface epidermal anomalies, such as altered dermatoglyphic lines and extranumerary nipples $(2,18)$.

\section{Dermatoglyphic marks}

Dermatoglyphs is the term used to describe epidermal lines of the skin of digital pulps, palm of the hands and feet. Epidermal lines are already well developed around the second trimester, but the process is not concluded until the last trimester. Once complete, the lines remain unchanged until the end of an individual's life, except for growth in size (19). In 1939, DM have been described in Down syndrome patients (17). Later, in 1966, unusual DM were summarized in 68 clinical diseases, including chromosomal aberrations, monogenic diseases, and also diseases of uncertain genetic origin, including congenital heart disease, psoriasis, and alterations due to toxic environmental factors such as thalidomide and rubella (20-22). Neurological and epidermal tissues share some common aspects of development 
such as similar ectodermal origin, rapid development during the second trimester of gestation and susceptibility to neuronal growth factor. Cerebral and epidermal growth are both influenced by hormones like testosterone, in both sexes, during dermatoglyphic development. It is well known that epidermal growth factor stimulates astrocyte division after the beginning of differentiation of glial cells at 12 weeks of gestation and that these emerging glial cells have an important role in blood-brain barrier definition (23). DM become especially interesting in the study of association of problems of cerebral development involving several neurological disorders in twins discordant for schizophrenia. These include hippocampal-amygdala complex reduction, ventricular dilatation and high scores of neurological alterations in the affected twins. This evidence has encouraged the search for environmental factors acting as "genotypic liberators" (19,24-27). Bracha (25), in a study of monozygotic twins discordant for schizophrenia, confirmed changes in dermatoglyphic counts, such as had previously been described in schizophrenic patients, providing further evidence of some deleterious effects around the sixth month of pregnancy leading to simultaneous neurologic and dermatologic abnormalities. It is important to point out that monozygotic and dizygotic twins do not necessarily share the same prenatal environment. One of the great determinants of the prenatal environment is the placenta. Approximately a third of monozygotic twins and all dizygotic twins are dichorionic. Davis et al. (19) proposed that variations in twin placentations also account for differences in twin concordance, even for schizophrenia, when it is considered that prenatal infections play a role in its etiology $(19,25,28,29)$.

\section{Minor physical anomalies}

Individual physical alterations are not very important from a clinical or cosmetic point of view, and it is acknowledged that normal people can have one to four of these anomalies. An excessive number of MPAs, however, is associated with several childhood behavioral problems and suggests genetic neurodevelopmental influences. Waldrop et al. (30) elaborated a scale for 18 different abnormalities associated with the first trimester of development involving head, hands and feet. These include low ear implantation, hypertelorism, high-arched palate and microcephalus, among others. Several neurological disorders like epilepsy, mental retardation and learning disabilities are associated with the presence of these anomalies. Studies comparing schizophrenic patients with normal controls also found an excess of MPAs in schizophrenics $(15,16)$. In a study correlating MPAs with Wechsler Scale performance a strong correlation of MPAs with lower verbal IQ and poor premorbid adjustment was observed (16). The authors suggest that this finding can indicate first-trimester insults leading simultaneously to MPAs and neurological abnormalities and consequently to impaired social adaptation and, perhaps, schizophrenia (16).

In any speculation about the role of MPA in schizophrenia, it is important to keep in mind that MPAs are nonspecific predictors of potential psychopathology in schizophrenia, evidencing disruption of normal firstand second-trimester gestation. Green et al. (15), in a non-controlled study, found MPAs associated with early onset of illness (before age 18). A later study comparing patients with schizophrenic and bipolar disease and family controls found increased prevalence of MPAs among schizophrenic patients, confirming previous associations of these markers with earlier age of onset and lower premorbid adjustment. The authors suggest that MPA may be predominantly associated with congenital rather than genetically determined cases of schizophrenia, linking prenatal insults to increased risk for schizophrenia (15). However, at least one study has 
demonstrated high rates of MPAs in the relatives of schizophrenic probands, suggesting a genetic association (18).

\section{Risk factors}

Countless factors have been associated with increased risk for schizophrenia, including maternal malnutrition or intoxication, infections, obstetric complications, maternal stress, in addition to different genetic factors. The genetic factors are the most robust of all, contributing 30 to $40 \%$ of the agreement observed in monozygotic twins (31). This partial agreement in monozygotic twins provides additional support to the most accepted hypothesis of a multifactorial synergism in a threshold model for schizophrenia (32-42).

\section{O bstetric complications}

There are several lines of evidence suggesting that obstetric complications are important risk factors for at least some types of schizophrenia. The concept of obstetric complications is, however, broad enough to allow different meanings. It represents a variety of different somatic deviations with probably different risks for schizophrenia (38). Schizophrenic patients at high genetic risk with a history of serious childbirth complications, anoxia, secondary hemorrhage and birth complications are at increased risk to display enlarged third ventricle, periventricular damage, and prevalence of negative symptoms (43). Geddes (44), in a meta-analysis of 12 studies looking for complications of pregnancy and labor in 700 schizophrenics and 635 controls, found a significant association between schizophrenia and premature rupture of membranes, gestational age less than 37 weeks and use of resuscitation or of an incubator. They also found a borderline association with forceps delivery and birth weight lower than $2,500 \mathrm{~g}$.

Some obstetric complications originate during the prenatal period, and several authors have suggested that this might well be a consequence, rather than a cause of the disease, indicating that fetuses at higher genetic risk for schizophrenia with intrauterine damage are at increased risk for asphyxia and anomalous presentations, in the same way as malformed fetuses (45). Jones et al. (46), in a North Finland 28-year cohort study, found associations between low birth weight (defined as less than 2,500 g) combined with prematurity (less than 37 weeks) and later schizophrenia, also indicating problems in fetal development. McNeil and Cantor-Graae (47) did not find support for this association in a recent study comparing schizophrenic, discordant and concordant monozygotic twins for schizophrenia and individuals at genetic risk. They suggested that complications occurring at labor-delivery do not represent an epiphenomenal consequence of preexisting fetal abnormality and are rather an etiological factor.

Some studies have suggested a possible connection between obstetric complications and ventricular enlargement but others have failed to confirm it (2). It has also been suggested that males are more sensitive to obstetric complications than females, probably due to differences in timing of cerebral maturation $(43,45)$.

\section{Maternal nutrition}

There is increased evidence concerning the association of nutritional factors and schizophrenia. Brown et al. (35), in a recent review, concluded that the effects of food deprivation are compatible with increased rates of schizophrenia and that food deprivation causes adverse neurodevelopmental effects. To explain the small impact on frequency distribution in countries with endemic famine they emphasized the fact that in these countries chronic malnutrition is often accompanied by other long-standing health and social problems such as infec- 
tions and poor prenatal and postnatal care. This, in turn, could decrease the chances of survival of fetuses and newborns until the age of risk for schizophrenia. The authors also suggest that deficiency of specific nutrients causing neural tube defects have been reported even in countries with adequate food supplies. Susser and Lin (48), in a retrospective study of a Dutch population submitted to food deprivation during the winter of 1944-45, found a larger number of psychiatric admissions for schizophrenia 20 years later, suggesting that food deprivation during pregnancy increased the risk for schizophrenia.

In spite of these observations, the only definitive evidence is the association of lowered ingestion of folic acid during the first trimester of pregnancy with an increased risk for neural tube defects (spina bifida, hydrocephalus, anencephaly). Evidence relating nutritional deprivation in utero to subsequent schizophrenia can be considered to be a strong argument favoring the retinoid theory of schizophrenia developed by Goodman $(18,49)$.

\section{Seasonality}

Epidemiological studies in temperate countries have pointed to the increased prevalence of births of schizophrenics in late winter and early spring. Similar, but less pronounced increases have been reported for bipolar disorder, but not for neurotic or personality disorders. Fanãnas et al. (50) have suggested that higher rates of winter and spring births could result from seasonal variation of parental procreational habits that may be age and class associated, but not associated with variables related to the disease itself. However, subsequent studies failed to confirm this explanation (51).

Much evidence links seasonal atmosphere changes with fetal damage, growth retardation and increased risk for schizophrenia (38). Environmental factors suggesting the association of birth seasonality with schizophrenia include infectious agents, nutritional factors and environmental temperature changes during childbirth. Crow (52) argued for an interaction of these factors with genetic ones. Among these factors, prenatal viruses are among the most common explanations.

Many infectious diseases show a seasonal pattern, with typical peaks of incidence. Rubella, for example, peaks in the autumn, following maternal infection during spring (peak season for adult rubella). The seasonality for anencephaly, congenital abnormalities and mental retardation is explained in the same way. However, seasonal changes or any other environmental changes contributing to the etiology of schizophrenia may not necessarily occur during the prenatal period (38). All the above-mentioned factors provide clues pointing to altered biochemistry in schizophrenia. This happens in primates mainly because of the maturational delay of the central nervous system (CNS), with brain development continuing into early and late childhood. This results in several critical periods over many months and years after birth. This long developmental timetable contributes to the chance of increased CNS vulnerability to negative environmental effects.

\section{Infectious diseases}

Several epidemiological studies have shown that maternal exposure to influenza virus or other viruses during the second trimester of gestation is associated with increased incidence of schizophrenia later in adulthood. There is still controversy about the role of viruses in the etiology of schizophrenia. This is due to the lack of direct evidence for this association, which could be demonstrated by the identification of an antiviral antibody or virus itself in serum, fluid or cerebral tissue of schizophrenic patients. An additional difficulty in the confir- 
mation of this theory derives from the long period of time from gestational exposure to outcome (20 or 30 years later) $(32-34,36,43$, 53-56).

Wright and Murray (57) reviewed the association of prenatal influenza and autoimmunity in schizophrenia. The authors cited Esquirol who, in 1838, noticed "epidemics" of psychotic disorders he called "insanity" involving large numbers of individuals. Also, Kraeplin described a "precocious" insanity and hypothesized that infections "in developmental years" could have some causal role. This idea was later reinforced by the endemic nature of several bacterial infections at the beginning of this century, like syphilis, tuberculosis, typhoid fever, cholera and influenza after the 1917 epidemic $(52,57)$. Still, the mechanism of viral CNS damage has not been well described. It is known that several neurotrophic viruses like influenza, Epstein-Barr or cytomegalovirus can cross the blood-brain barrier. In the case of influenza this can occur through the expression of capsular neuraminidase that alters "sialic moiety acids", enzymes of neural adhesion molecules that regulate neuronal migration during neuroembryogenesis $(38,57)$. It is also possible that maternal virus antibodies act as fetal anti-cerebral antibodies, since these antibodies are known to cross the placenta. It is also possible that some unspecific factors of infection like fever or anoxia could account for CNS pathology (38).

Recent population studies evaluating the influenza epidemics of 1954, 1957 and 1959 in Australia and Japan have revealed positive associations between gestational exposure to virus and later schizophrenia. The Japanese study found an increased risk for female schizophrenia. McGrath et al. (54) suggested a difference related to the month of viral exposure (excess of male schizophrenia births 4 months after inception of the 1954 epidemic and of female births 5 months after the 1957 epidemic, with no association in the 1959 epidemic) $(53,54)$.

A factor confounding a viral causation may be the maternal use of analgesics to treat viral infection during gestation. Use of analgesics has been associated with increased risk of anencephaly and other congenital CNS defects. Acetylsalicylic acid is a potent anticoagulant that can increase fetal brain hemorrhages. The association between decreased aspirin use in Finland and Scandinavia in the sixties and reduced incidence of schizophrenia later in the 1990's (38) still needs confirmation by additional studies.

\section{Final considerations}

The present review shows that in a large number of cases of schizophrenia there is consistent evidence supporting a combined effect of genetic and congenital factors generating neurodevelopmental abnormalities. These, in turn, could lower the threshold for schizophrenia through several biochemical and physiologic mechanisms, with faulty "thalamic filtering", lowering "buffering capacity" for stress control, and other feedback mechanisms for environmental adjustment. More detailed tests of this theory using various combinations of specific risk factors invoking the threshold model of schizophrenia are needed to describe the full range of neurodevelopmental abnormalities in the disease. Neuronal migration abnormalities in schizophrenia have been associated with maternal famine (48), and more recently with retinoid dysregulation $(18,49)$. A precise identification of these mechanisms under the neurodevelopmental theory of schizophrenia would allow a better understanding of the physiopathology and biochemistry of the disease and also permit a better risk assessment and early intervention in different stages of the disorder. Furthermore, understanding causation in schizophrenia could provide avenues for specific interventions for high-risk subjects and preventive correction of altered nutritional or biochemical 
defects. This is the case in possible retinoid cascade dysregulation $(18,49)$ and in altered expression of phospholipids (58) or ectoenzymes (5'-nucleotidase) (59). These possibilities, if confirmed according to the neurodevelopmental theory of schizophrenia and related illnesses, would pave the road for specific and potent treatments for these catastrophic disorders.

\section{Acknowledgments}

We thank Ann Goodman for the careful review and suggestions.

\section{References}

1. Bogerts B (1993). Recent advances in the neuropathology of schizophrenia. Schizophrenia Bulletin, 19: 431-445.

2. O'Connell P, Woodruff PW, Wright I, J ones P \& Murray RM (1997). Developmental insanity or dementia praecox: was the wrong concept adopted? Schizophrenia Bulletin, 23: 97-106.

3. Stevens JR (1997). Anatomy of schizophrenia revisited. Schizophrenia Bulletin, 23: 373-383.

4. Davidson $M$, Reichenberg $A$, Rabinowitz J , Weiser M, Kaplan Z \& Mark M (1999). Behavior and intellectual markers for schizophrenia in apparently healthy male adolescents. American J ournal of Psychiatry, 156: 1328-1335.

5. Rakic P (1988). Defects of neuronal migration and the pathogenesis of cortical malformations. Progress in Brain Research, 73: 15-37.

6. Powchik P, Davidson M, Haroutunian V, Gabriel SM, Purohit DP, Perl DP, Harvey PD \& Davis KL (1998). Postmortem studies in schizophrenia. Schizophrenia Bulletin, 24: 325-341.

7. Nelson MD, Saykin AJ, Flashman LA \& Riordan HJ (1998). Hippocampal volume reduction in schizophrenia as assessed by magnetic resonance imaging: a meta-analytic study. Archives of General Psychiatry, 55: 433-440.

8. Akbarian S, Vinuela A, Kim J J , Potkin SG, Bunney J r WE \& J ones EG (1993). Distorted distribution of nicotinamide-adenine dinucleotide phosphate-diaphorase neurons in temporal lobe of schizophrenics implies anomalous cortical development. Archives of General Psychiatry, 50: 178-187.

9. Chua SE \& McKenna PJ (1995). Schizophrenia - a brain disease? A critical review of structural and functional cerebral abnormality in the disorder. British J ournal of Psychiatry, 166: 563-582.

10. Gur RE, Turetsky BI, Bilker WB \& Gur RC
(1999). Reduced gray matter volume in schizophrenia. Archives of General Psychiatry, 56: 905-911.

11. Abreu P, Busnello E, Chaves M, llha D, Baron A, Camozzatto A, Lobato $M$ \& Schestatsky G (1995). Case-control study of tomographic alterations in schizophrenia. Revista de Psiquiatria do Rio Grande do Sul, 17: 112-120.

12. Illowsky B, Juliano DM, Bigelow LB \& Weinberger DR (1988). Stability of CT scan findings in schizophrenia: results of an 8-year follow-up study. J ournal of Neurology, Neurosurgery and Psychiatry, 51: 209-213.

13. Cannon $M$, Byrne $M$, Cotter $D$, Sham $P$, Larkin C \& O'Callaghan E (1994). Further evidence for anomalies in the handprints of patients with schizophrenia: a study of secondary creases. Schizophrenia Research, 13: 179-184.

14. Fañanas $\mathrm{L}, \mathrm{M}$ oral $\mathrm{P} \&$ Bertranpetit J (1990). Quantitative dermatoglyphics in schizophrenia: study of family history subgroups. Human Biology, 62: 421-427.

15. Green MF, Satz P, Gaier DJ , Ganzell S \& Kharabi $F$ (1989). Minor physical anomalies in schizophrenia. Schizophrenia Bulletin, 15: 91-99.

16. Guy JD, Majorski LV, Wallace CJ \& Guy MP (1983). The incidence of minor physical anomalies in adult male schizophrenics. Schizophrenia Bulletin, 9: 571-582.

17. Varma SL, Chary TV, Singh S, Azhar MZ \& Dharap AS (1995). Dermatoglyphic patterns in schizophrenic patients. Acta Psychiatrica Scandinavica, 91: 213-215.

18. Goodman A (1996). Congenital anomalies in relatives of schizophrenic probands may indicate a retinoid pathology. Schizophrenia Research, 19: 163-170.

19. Davis J O, Phelps J A \& Bracha HS (1995). Prenatal development of monozygotic twins and concordance for schizophrenia. Schizophrenia Bulletin, 21: 357-366.

20. Achs R, Harper RG \& Siegel M (1966).
Unusual dermatoglyphic findings associated with rubella embryopathy. New England J ournal of Medicine, 274: 148-150.

21. Holt SB (1961). Quantitative genetics of fingerprint patterns. British Medical Bulletin, 17: 247-250.

22. Purvis-Smith SG \& Menser MA (1973). Genetic and environmental influences on digital dermatoglyphics in congenital rubella. Pediatric Research, 7: 215-219.

23. Patterson PH (1995). Neuronal growth and differentiation factors and synaptic plasticity. In: Bloom FE \& Kupper DJ (Editors), Psychopharmacology: the Fourth Generation of Progress. Raven Press, New York.

24. Belmaker $\mathrm{R}$, Pollin $\mathrm{W}$, Wyatt $\mathrm{RJ} \&$ Cohen $S$ (1974). A follow-up of monozygotic twins discordant for schizophrenia. Archives of General Psychiatry, 30: 219-222.

25. Bracha HS (1991). Etiology of structural asymmetry in schizophrenia: an altemative hypothesis. Schizophrenia Bulletin, 17: 551-553.

26. Davis J O \& Bracha S (1996). Prenatal growth markers in schizophrenia: the monozygotic co-twin control study. American J ournal of Psychiatry, 153: 1166-1171.

27. LohrJ B \& Bracha HS (1992). The monozygotic mirror-image twin pairs with discordant psychiatric illnesses: the neuropsychiatric and neurodevelopmental evaluation. American J ournal of Psychiatry, 149: 1091-1095.

28. Bracha HS, Torrey EF, Gottesman II, Bigelow LB \& Cunniff C (1992). Secondtrimester markers of fetal size in schizophrenia: the study of monozygotic twins. American J ournal of Psychiatry, 149: 1355-1360.

29. Bracha HS, Torrey EF, Bigelow LB, Lohr J B \& Linington BB (1991). Subtle signs of prenatal maldevelopment of the hand ectoderm in schizophrenia: a preliminary monozygotic twin study. Biological Psychiatry, 30: 719-725.

30. Waldrop M, Pederson F \& Bell R (1968). 
Minor physical anomalies and behavior in preschool children. Child Development, 39: 391-400.

31. Torrey EF (1992). Are we overestimating the genetic contribution to schizophrenia? Schizophrenia Bulletin, 18: 159-170.

32. Adams W, Kendell RE, Hare EH \& MunkJ orgensen $P$ (1993). Epidemiological evidence that maternal influenza contributes to the etiology of schizophrenia. An analysis of Scottish, English, and Danish data. British J ournal of Psychiatry, 163: 522534.

33. Adams W \& Kendell RE (1996). Influenza and schizophrenia (Comments). British J ournal of Psychiatry, 169: 252-253.

34. Barr CE, Mednick SA \& Munk-J orgensen $P$ (1990). Exposure to influenza epidemics during gestation and adult schizophrenia. Archives of General Psychiatry, 47: 869-874.

35. Brown A, Susser E, Butler P, Andrews R, Kaufmann C \& Gorman J (1996). Neurobiological plausibility of prenatal nutritional deprivation as a risk factor for schizophrenia. J ournal of Nervous and Mental Disease, 184: 71-85.

36. Cannon M, Cotter D, Coffey VP, Sham P, Takei N, Larkin C, Murray RM \& O'Callaghan E (1996). Prenatal exposure to the 1957 influenza epidemic and adult schizophrenia: a follow-up study. British J oumal of Psychiatry, 168: 368-371.

37. Gottesman II \& Bertelsen A (1989). Confirming unexpressed genotypes for schizophrenia. Archives of General Psychiatry, 46: 867-872.

38. Huttunen MO, Machon RA \& Mednick AS (1994). Prenatal factors in the pathogenesis of schizophrenia. British J ournal of Psychiatry, 164 (Suppl 23): 15-19.

39. J ones P \& Murray R (1991). The genetics of schizophrenia is the genetics of neurodevelopment. British J ournal of Psychiatry, 158: 615-623.

40. Moldin SO \& Gottesman II (1997). At is- sue: genes, experience, and chance in schizophrenia positioning for the 21st century. Schizophrenia Bulletin, 23: 547-561.

41. Murphy KC \& Owen MJ (1996). Schizophrenia, CATCH 22 and FISCH. British J ournal of Psychiatry, 168: 397-398.

42. Myhrman A, Rantakallio $P$, Isohanni $M$, J ones P \& Partanen U (1996). Unwantedness of a pregnancy and schizophrenia in the child. British J ournal of Psychiatry, 169: 637-640.

43. Wright $\mathrm{T}$, Takei N, Rifkin L \& Murray RM (1995). Maternal influenza, obstetric complications and schizophrenia. American J ournal of Psychiatry, 152: 1714-1720.

44. Geddes J R (1999). Schizophrenia and complications of pregnancy and labor: an individual patient data meta-analysis. Schizophrenia Bulletin, 25: 413-423.

45. Cantor-Graae E, McNeil TF, Rickler KC, Sjostrom K, Rawlings R, Higgins ES \& Hyde TM (1994). Are neurological abnormalities in well discordant monozygotic co-twins of schizophrenic subjects the result of perinatal trauma? American J ournal of Psychiatry, 151: 1194-1199.

46. J ones PB, Rantakallio P, Hartikainen A, Isohanni M \& Sipila P (1998). Schizophrenia as a long term outcome of pregnancy, delivery, and perinatal complications: a 28year follow-up of the 1966 North Finland general population birth cohort. American J ournal of Psychiatry, 155: 355-364.

47. McNeil T \& Cantor-Graae E (1999). Does preexisting abnormality cause labor-delivery complications in fetuses who will develop schizophrenia? Schizophrenia Bulletin, 25: 425-435.

48. Susser E \& Lin SP (1992). Schizophrenia after prenatal exposure to the Dutch hunger winter of 1944-1945. Archives of General Psychiatry, 49: 983-988.

49. Goodman A (1998). Three independent lines of evidence suggest retinoids as causal to schizophrenia. Proceedings of the National Academy of Sciences, USA,
95: 7240-7244.

50. Fañanas $L$, Marti-Tusquets J L \& Bertranpetit J (1989). Seasonality of birth in schizophrenia. An insufficient stratification of control population? Social Psychiatry and Psychiatric Epidemiology, 24: 266270.

51. Torrey EF \& Rawlings RR (1996). Fluctuations in schizophrenic births by year. British J ournal of Psychiatry, 169: 772-775.

52. Crow TJ (1994). Prenatal exposure to influenza as a cause of schizophrenia: there are inconsistencies and contradictions in the evidence. British J ournal of Psychiatry, 164: 588-592.

53. Kunugi $\mathrm{H}$, Nanko S, Takei N, Saito $\mathrm{K}$, Hayashi N \& Kazamatsuri H (1995). Schizophrenia following in utero exposure to the 1957 influenza epidemics in J apan. American J ournal of Psychiatry, 152: 450-452.

54. McGrath J, Pemberton MR, Welham J L \& Murray RM (1994). Schizophrenia and the influenza epidemics of 1954, 1957 and 1959: a southern hemisphere study. Schizophrenia Research, 14: 1-8.

55. Mednick AS, Machon RA, Huttunen MO \& Bonett D (1988). Adult schizophrenia following prenatal exposure to an influenza epidemic. Archives of General Psychiatry, 45: 189-192.

56. Takei $\mathrm{N}$, Lewis $\mathrm{S}$, J ones $\mathrm{P}$, Harvey I \& Murray RM (1996). Prenatal exposure to influenza and increased cerebrospinal fluid spaces in schizophrenia. Schizophrenia Bulletin, 22: 521-534.

57. Wright $P \&$ Murray RM (1993). Schizophrenia: prenatal influenza and autoimmunity. Annals of Medicine, 25: 497-502.

58. Horrobin DF (1998). The membrane phospholipid hypothesis as a biochemical basis for neurodevelopmental concept of schizophrenia. Schizophrenia Research, 30: 193-208.

59. Lara DR \& Souza DO (2000). Schizophrenia: a purinergic hypothesis. Medical Hypotheses, 54: 157-166. 